\title{
RAFT "grafting-through" approach to surface-anchored polymers: Electrodeposition of an electroactive methacrylate monomer
}

\author{
C.D. Grande ${ }^{1,2}$, M.C. Tria ${ }^{1}$, M.J. Felipe ${ }^{1}$, F. Zuluaga ${ }^{2}$, and R. Advincula ${ }^{1, a}$ \\ 1 Department of Chemistry and Department of Chemical and Biomolecular Engineering, University of Houston, Houston, TX \\ 77204-5003, USA \\ 2 Departamento de Quimica, Universidad del Valle, A.A. 25360, Cali, Colombia
}

Received 29 July 2010

Published online: 21 February 2011

(c) The Author(s) 2011. This article is published with open access at Springerlink.com

\begin{abstract}
The synthesis of homopolymer and diblock copolymers on surfaces was demonstrated using electrodeposition of a methacrylate-functionalized carbazole dendron and subsequent reversible additionfragmentation chain transfer (RAFT) "grafting-through" polymerization. First, the anodically electroactive carbazole dendron with methacrylate moiety (G1CzMA) was electrodeposited over a conducting surface (i.e. gold or indium tin oxide (ITO)) using cyclic voltammetry (CV). The electrodeposition process formed a crosslinked layer of carbazole units bearing exposed methacrylate moieties. This film was then used as the surface for RAFT polymerization process of methyl methacrylate (MMA), styrene (S), and tert-butyl acrylate (TBA) in the presence of a free RAFT agent and a free radical initiator, resulting in grafted polymer chains. The molecular weights and the polydispersity indices (PDI) of the sacrificial polymers were determined by gel permeation chromatography (GPC). The stages of surface modification were investigated using X-ray photoelectron spectroscopy (XPS), ellipsometry, and atomic force microscopy (AFM) to confirm the surface composition, thickness, and film morphology, respectively. UV-Vis spectroscopy also confirmed the formation of an electro-optically active crosslinked carbazole film with a $\pi-\pi^{*}$ absorption band from 450-650 nm. Static water contact angle measurements confirmed the changes in surface energy of the ultrathin films with each modification step. The controlled polymer growth from the conducting polymer-modified surface suggests the viability of combining electrodeposition and grafting-through approach to form functional polymer ultrathin films.
\end{abstract}

\section{Introduction}

Ultrathin film coatings applied onto surfaces can improve surface properties of bulk materials or can even prevent damage from the environment by controlling the interaction of the surface from outside conditions. Functional ultrathin film coatings have become an enabling technology for various applications [1]. Fields in which smart or stimuli-responsive coatings are applied range from computer chips [2], hard disk manufacturing [3], to biomedical and aviation applications $[4,5]$. Many different techniques have been developed for the fabrication of protective and nanostructured coatings such as spin-coating [6], dip-coating [7], layer-by-layer [8], and drop casting [9]. However in these processes, the physically adsorbed polymer films can be easily desorbed or delaminated from the surface under unfavorable conditions.

\footnotetext{
${ }^{a}$ e-mail: radvincula@uh.edu
}

"Grafting to" [10] and "grafting from" (or surfaceinitiated polymerization (SIP)) [11,12] have also become popular techniques in preparing polymer coatings on surfaces. These techniques are based on tethering polymers on surfaces by either using a preformed polymer with an active end group to attach to the surface, in the case of "grafting to" technique, or by growing polymers in situ from surface-bound initiators, in the case of SIP [1]. In this way, the chemical attachment of the polymers on the substrate provides a more robust and good long-term stability, which are highly desirable for coatings.

Another straightforward technique for the attachment of polymers to surfaces is to carry out a polymerization reaction in the presence of a substrate with tethered monomers [13-18]. In such polymerization reaction, the surface-attached monomers are incorporated into the growing polymer chains in a "grafting-through" manner, where the polymers are eventually bound to the surface after removal of excess physically adsorbed polymers. This approach is one of the simple techniques for generating 
surface-attached polymer layers, especially as there is no need to synthesize preformed polymers with an anchoring group ("grafting to") or deal with the mechanistic difficulties of SIP ("grafting from") [1].

Electropolymerization of heteroaromatic monomers such as thiophene, pyrrole, aniline, and carbazole to form $\pi$-conjugated or conducting polymers has been well documented [19-22] The study of such electropolymerizable monomers is interesting, as it can yield unique polymerization mechanisms and electro-optical applications including electrochromic properties [23]. In our group, carbazolecontaining polymers and dendrimers have been synthesized and studied [24-28] because of their potential applications on electroluminescent devices [29-31], photorefractive materials [32,33], electrochromic materials [34, $35]$, and memory devices [36]. In all the above applications, the main use of carbazole-derivatized polymers for electro-optical function involves efficient hole transport, good solution processing properties, and high chemical stability [37-40]. Using the precursor polymer approach, polymers with electroactive side-chain functional groups can form conjugated polymer networks ( $\mathrm{CPNs}$ ) on the electrode surface upon chemical or electrochemical oxidation processes $[24-28,37]$. The CPN formation [41,42] has also been applied to dendrimeric precursors forming shape-persistent conjugated nano-objects and electrooptically active films $[43,44]$.

In this study, we have combined the "graftingthrough" approach and the formation of CPN via the electrodeposition of a first-generation carbazole dendron bearing a methacrylate group at the focal point, G1CzMA, onto a conducting surface such as gold or ITO. In particular, we used the RAFT technique for the polymerization process because of its living and controlled characteristics, as well as its versatility to a wide range of monomers [45]. This tandem approach offers an advantage of fabricating films with dual (CPN and homopolymer brush) or multi-component (CPN and block copolymers) polymer system. Furthermore, the use of an electrochemically active monomer enables its deposition on any conducting surfaces. The electrodeposition of the precursor G1CzMA was done by cyclic voltammetry (CV) to enable anodic electropolymerization and crosslinking of the carbazole moieties. The resulting electro-optically active layer is employed for grafting polymers onto the surface in a "grafting-through" manner by exposing the substrate to a solution of the monomer (methyl methacrylate (MMA), styrene (S), or tert-butyl acrylate (TBA)), initiator and the RAFT agent. To the best of our knowledge, this represents the first report on the synthesis of RAFT grafted polymers using an electrochemically anchored carbazole dendron bearing methacrylate moiety on electrode surfaces.

\section{Experimental}

\subsection{Materials}

All materials and reagents were purchased from Aldrich, VWR or Acros Organics and were used without fur- ther purification unless otherwise indicated. Tetrahydrofuran (THF) used in the syntheses was distilled from sodium benzophenone ketyl. Styrene, methyl methacrylate, and tert-butyl acrylate were passed through a column with alternating layers of inhibitor remover and activated neutral alumina to remove the inhibitor. 2,2azobis(isobutyronitrile) (AIBN) was recrystallized twice from ethanol. Carbazole was recrystallized twice from methanol.

\subsection{Synthesis of 9-(4-Bromobutyl)-9H-carbazole [Cz-Br] (1)}

The synthesis of 9-(4-bromobutyl)-9H-carbazole was accomplished by a modified method as reported by Bo et al. [46]. A mixture of carbazole $(10.32 \mathrm{~g}, 61.72 \mathrm{mmol})$ in toluene $(100 \mathrm{~mL})$ containing 1,4-dibromobutane (118.2 g, $547.4 \mathrm{mmol}$ ) and tetrabutylammonium bromide (TBAB) $(2 \mathrm{~g}, 6.20 \mathrm{mmol})$ was stirred at $45^{\circ} \mathrm{C}$ for $3 \mathrm{~h}$ and was left overnight. After the aqueous layer was removed and washed three times with water and brine, the organic layer was dried over $\mathrm{Na}_{2} \mathrm{SO}_{4}$. The organic solvent was evaporated by rotavap while the unreacted 1,4-dibromobutane was removed by vacuum distillation. The residue was recrystallized from ethanol to give $16.7 \mathrm{~g}(89.5 \%)$ of the product. The characterization was found to be consistent with the literature.

\subsection{Synthesis of Methyl 3,5-Bis(4-(9H-carbazol-9-yl)butoxy)benzoate [G1Cz-COOR] (2)}

In a literature modified process as reported by $\mathrm{Fu}$ et al. [47], a mixture of $\mathrm{Cz}-\mathrm{Br}$ (1) (15.22 g, $50.37 \mathrm{mmol}), \mathrm{K}_{2} \mathrm{CO}_{3}(21.0 \mathrm{~g}, 151.94 \mathrm{mmol})$, methyl 3,5-dihydroxybenzoate $(3.94 \mathrm{~g}, 23.4 \mathrm{mmol}), 18$-crown-6 (45 mg, $0.170 \mathrm{mmol})$, and acetone $(500 \mathrm{~mL})$ was stirred and refluxed under nitrogen atmosphere for $72 \mathrm{~h}$. Acetone was evaporated under vacuum, and the residue was partitioned between water $(400 \mathrm{~mL})$ and $\mathrm{CH}_{2} \mathrm{Cl}_{2}(500 \mathrm{~mL})$. The organic layer was separated, and the aqueous layer was extracted three times with $\mathrm{CH}_{2} \mathrm{Cl}_{2}(250 \mathrm{~mL})$. Finally, the combined organic layer was dried over $\mathrm{Na}_{2} \mathrm{SO}_{4}$. After removal of the solvent, the residue was recrystallized from ethyl acetate to give a white solid product in $70 \%$ yield. The characterization data were found to be consistent with the literature.

\subsection{Synthesis of (3,5-Bis(4-(9H-carbazol-9-yl)butoxy)phenyl)metanol [G1Cz-OH] (3)}

Compound 2 (9.5 g, $15.5 \mathrm{mmol})$ dissolved in dry THF $(400 \mathrm{~mL})$ was added drop-wise to a suspension of lithium aluminum hydride $\left(\mathrm{LiAlH}_{4}\right)(1.0 \mathrm{~g}, 23.3 \mathrm{mmol})$ in THF $(300 \mathrm{~mL})$ cooled to $0{ }^{\circ} \mathrm{C}$ with an ice bath. The suspension was then allowed to warm to room temperature and 
was stirred until the reaction was complete as indicated by TLC. The reaction was quenched by adding water, and the THF was removed under reduced pressure in a rotary evaporator. The resulting layer was brought to a neutral $p \mathrm{H}$ with the addition of a $2 \mathrm{~N} \mathrm{HCl}$ solution and extracted with $\mathrm{CH}_{2} \mathrm{Cl}_{2}$. The organic layers were combined, washed with water, dried over sodium sulfate, filtered, and concentrated under reduced pressure. The product was purified by silica gel flash column chromatography using a 20:4:1 $\mathrm{CH}_{2} \mathrm{Cl}_{2}$ :hexane:ethyl acetate eluent to afford a white solid in $90 \%$ yield. ${ }^{1} \mathrm{H} \mathrm{NMR}\left(\mathrm{CDCl}_{3}\right): \delta(\mathrm{ppm}) 8.10(\mathrm{~d}, 4 \mathrm{H}$, $J=7.5 \mathrm{~Hz}), 7.53-7.42(\mathrm{~m}, 8 \mathrm{H}), 7.25-7.22(\mathrm{~m}, 4 \mathrm{H}), 6.45$ $(\mathrm{d}, 2 \mathrm{H}, J=2.1 \mathrm{~Hz}), 6.28(\mathrm{t}, 1 \mathrm{H}, J=2.1 \mathrm{~Hz}), 4.59(\mathrm{~s}, 2 \mathrm{H})$, $4.39(\mathrm{t}, 4 \mathrm{H}, J=6.9 \mathrm{~Hz}), 3.91(\mathrm{t}, 4 \mathrm{H}, J=6.2 \mathrm{~Hz}), 2.02-$ $2.12(\mathrm{~m}, 4 \mathrm{H}), 1.87-1.78(\mathrm{~m}, 4 \mathrm{H}) .{ }^{13} \mathrm{C} \mathrm{NMR}\left(\mathrm{CDCl}_{3}\right): \delta$ (ppm) 160.26, 144.33, 140.38, 128.4, 125.7, 122.89, 120.44, $118.89,108.69,105.19,100.57,67.58,65.34,42.75,27.02$, 25.91. Anal. Calcd: C, 80.38; H, 6.57; N, 4.81. Found: C, $80.12 ; \mathrm{H}, 6.44 ; \mathrm{N}, 4.95$.

\subsection{Synthesis of \\ 3,5-bis(4-(9H-carbazol-9-yl)butoxy)benzyl methacrylate (G1CzMA) (4)}

A solution of $\mathrm{G} 1 \mathrm{Cz}-\mathrm{OH}(3)(1.0 \mathrm{~g}, 1.72 \mathrm{mmol})$ and triethylamine (TEA) was combined in dichloromethane. A solution of methacryloyl chloride in dichloromethane was then added dropwise to the resulting mixture. The reaction mixture was stirred for 2 hours. The resulting solution was washed with water three times. The extract was then concentrated in vacuum and was purified by column chromatography using 4:1 $\mathrm{CH}_{2} \mathrm{Cl}_{2}$ : hexane as eluent. Yield: $50 \%{ }^{1} \mathrm{H} \mathrm{NMR}\left(\mathrm{CDCl}_{3}\right): \delta(\mathrm{ppm}) 8.12(\mathrm{~d}, 2 \mathrm{H}), 7.48-7.42$ $(\mathrm{m}, 8 \mathrm{H}), 7.28-7.22(\mathrm{~m}, 4 \mathrm{H}), 6.47(9 \mathrm{~m}, 2 \mathrm{H}), 6.34(\mathrm{~m}, 1 \mathrm{H})$, $6.16(\mathrm{~m}, 1 \mathrm{H}), 5.6(\mathrm{~m}, 1 \mathrm{H}), 5.1(\mathrm{~s}, 2 \mathrm{H}), 4.42(\mathrm{t}, 4 \mathrm{H}), 3.92$ $(\mathrm{t}, 4 \mathrm{H}), 2.12-2.07(\mathrm{~m}, 4 \mathrm{H}), 1.88-1.82(\mathrm{~m}, 4 \mathrm{H}) .(1.58 \mathrm{~g}) .{ }^{1} \mathrm{H}$ $8.11(\mathrm{~d}, 4 \mathrm{H}, J=7.8 \mathrm{~Hz}), 7.45-7.39(\mathrm{~m}, 8 \mathrm{H}), 7.25-7.19(\mathrm{~m}$, $4 \mathrm{H}), 6.45(\mathrm{~d}, 2 \mathrm{H}, J=2.4 \mathrm{~Hz}), 6.31(\mathrm{t}, 1 \mathrm{H}, J=2.1 \mathrm{~Hz})$, $6.14(\mathrm{~s}, 1 \mathrm{H}), 5.57(\mathrm{t}, 1 \mathrm{H}, J=1.2 \mathrm{~Hz}), 5.07(\mathrm{~s}, 2 \mathrm{H}), 4.39(\mathrm{t}$, $4 \mathrm{H}, J=6.9 \mathrm{~Hz}), 3.90(\mathrm{t}, 4 \mathrm{H}, J=6.2 \mathrm{~Hz}), 2.02-2.12(\mathrm{~m}$, $4 \mathrm{H}), 1.95(\mathrm{~s}, 3 \mathrm{H}), 1.87-1.78(\mathrm{~m}, 4 \mathrm{H}) .{ }^{13} \mathrm{C} \mathrm{NMR}\left(\mathrm{CDCl}_{3}\right)$ : $\delta(\mathrm{ppm}) 167.24,160.29,140.48,138.50,136.29,126.04$, $125.79,122.99,120.50,118.99,108.77,106.47,101.01$, 67.64, 66.34, 42.74, 27.05, 25.95, 18.50. Anal. Calcd: C, 79.36; H, 6.50; N, 4.30, O, 9.83. Found: C, 78.96; H, 6.64; $\mathrm{N}, 4.24, \mathrm{O}, 9.98$.

\subsection{Electrodeposition of the G1CzMA}

The formation of the electrochemically polymerized (crosslinked) G1CzMA film was done in a three-electrode cell. A solution of $0.1 \mathrm{M}$ tetrabutylammonium hexafluorophosphate (TBAH) as supporting electrolyte and $5 \mathrm{mM}$ of the G1CzMA in dry THF was used. After mixing and stirring, the voltage was swept at a scan rate of $100 \mathrm{mV} / \mathrm{s}$ from 0 to $1.2 \mathrm{~V}$ against $\mathrm{Ag} / \mathrm{AgCl}$ reference electrode and $\mathrm{Pt}$ counter electrode for 10 cycles. ITO and gold were used as the working electrodes.

\subsection{RAFT "grafting through" of the polymers on the surface}

For brevity, only the procedure for RAFT "grafting through" of PMMA polymer chains is described. For a typical run, the chain transfer agent, 2-(phenylcarbonothioylthio)acetic acid (CTA 1) (26.50 mg, $0.124 \mathrm{mmol}$ ), azobisisobutyronitrile (AIBN) (4.07 mg, $24.8 \mu \mathrm{mol})$, and MMA $(5.00 \mathrm{~g}, 49.94 \mathrm{mmol})$ were added to a $50 \mathrm{~mL}$ Schlenk flask. Polymerizations were performed under homogeneous conditions with $6 \mathrm{~mL}$ of dry THF: benzene (1:1) mixture as solvent. The monomer mixture was degassed by bubbling the system with pure nitrogen for 1 hour and was transferred to a second Schlenk tube containing the G1CzMA-modified ITO or gold substrate backfilled with nitrogen using a cannula. The flask with the substrate and monomer solution was placed in an oil bath at $60^{\circ} \mathrm{C}$. After 18 hours, the slide was removed and the free polymers were quenched to $0^{\circ} \mathrm{C}$. Untethered polymers were removed from the substrate via Soxhlet extraction overnight at $60^{\circ} \mathrm{C}$ in THF. Free polymers from the polymerization solutions were precipitated in hexane. This procedure was repeated until no monomer signals were observed in the ${ }^{1} \mathrm{H}-\mathrm{NMR}$. The resulting polymer was dried under vacuum at room temperature until no weight loss was observed and then analyzed by GPC. The same procedure was used for styrene (S) and tert-butyl acrylate (TBA) polymerizations where a mole ratio of 400:1:0.2 for monomer:CTA:AIBN was used.

\subsection{Formation of the diblock copolymers via RAFT polymerization}

The general procedure for the preparation of diblock copolymers from the substrate was similar to the described PMMA homopolymer preparation, but using either the PMMA-modified or PS-modified gold substrate instead. For example, a molar ratio between the styrene monomer and CTA 1 of 400:1 and CTA to initiator of 5:1 in a solution of THF was used. The solution was added via cannula to the second Schlenk tube containing the PMMAmodified substrate backfilled with nitrogen. The reaction was terminated after a specified period of time. The substrate was isolated and any physisorbed polymers were removed via Soxhlet extraction. The sacrificial polymers were obtained by precipitation from hexanes and were used to estimate the molecular weight and PDI of the second grafted polymer chains.

\subsection{Characterization}

Nuclear magnetic resonance (NMR) spectra were recorded on a General Electric QE-300 spectrometer at $300 \mathrm{MHz}$ for ${ }^{1} \mathrm{H}-\mathrm{NMR}$. UV-Vis spectra were recorded on an Agilent 8453 spectrometer. GPC was used to determine the molecular weight of the sacrificial polymers: Viscotek 270 instrument with a triple detector array (RALS, IV, RI, 
or UV) equipped with 2 GMHHR-M and 1 GMHHRL mixed bed ViscoGel columns (eluent: THF; flow rate: $\left.1 \mathrm{~mL} \mathrm{~min}{ }^{-1}\right)$. CV was performed on an Amel 2049 potentiostat and power lab/4SP system with a three-electrode cell. In all the measurements the platinum wire was used as the counter electrode, Ag wire as the reference electrode, and gold or ITO as the working electrodes. AFM imaging was performed under ambient conditions using a PicoScan 2500 (Agilent Technologies formerly Molecular Imaging). Intermittent contact mode was used for all imaging. All thickness measurements were conducted using a null ellipsometer operating in polarizer-compensator sample-analyzer (Multiskop, Optrel Berlin) mode. A HeNe laser $(\lambda=632.8 \mathrm{~nm})$ was used as the light source, and the angle of incidence was set to $70^{\circ}$. A multilayer flat Fresnel film model was used to calculate the thicknesses from the experimentally measured ellipsometric angles $\Delta$ and $\psi$, assuming a refractive index of 1.50 for the polymers [48]. The gold electrodes were cleaned with a plasma ion cleaner (Plasmod, March). XPS was carried out in a Physical Electronics 5700 instrument with photoelectrons generated by the nonmonochromatic $\mathrm{Al} K_{\alpha}$ irradiation $(1486.6 \mathrm{eV})$. Photoelectrons were collected at a takeoff angle of $45^{\circ}$ using a hemispherical analyzer operated in the fixed retard ratio mode with an energy resolution setting of $11.75 \mathrm{eV}$. The binding energy scale was calibrated prior to analysis using the $\mathrm{Cu} 2 p_{3 / 2}$ and $\mathrm{Ag} 3 d_{5 / 2}$ lines. Charge neutralization was ensured through bombardment of the irradiated area with an electron beam and the use of the non-monochromatic $\mathrm{Al} K_{\alpha}$ source. This placed the adventitious C $1 s$ peak at a binding energy of $284.6 \pm 0.2 \mathrm{eV}$. Static water contact angle goniometry was conducted using a KSV CAM 200 instrument (KSV Ltd.) using the bubble drop method with water.

\section{Results and discussion}

The use of controlled radical polymerization techniques or CLRP has received much attention for preparing surfacegrafted polymers [49]. This is due to the ability of the method to control polymerization over several monomers including styrenics, methacrylates and acrylates, and the possibility for block copolymerization. Together with good control over thickness and homogeneity over the surface, the possibility for complex architectures and compositions is very attractive [50]. Among the CLRP methods, RAFT polymerization offers many advantages for the synthesis of a large variety of complex and highly functionalized architectures [51]. In this study, RAFT "grafting-through" approach was employed wherein the surface with bound monomer molecules was placed in a solution where typical RAFT polymerization is conducted (fig. 1). Specifically, the monomer employed has an electroactive moiety that can be electrodeposited on the surface, which at the same time can produce a $\pi$-conjugated layer. This approach has a distinct possibility of finding future applications for ultrathin films with electro-optical properties combined with the properties of the grafted polymers. From the viewpoint of the conjugated polymer, this produces a main-chain conjugated polymer backbone with a highly CPN.

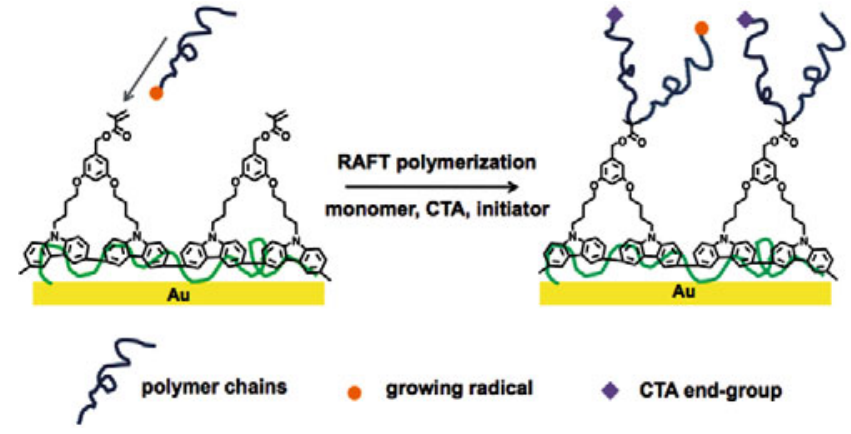

Fig. 1. An ideal representation of the RAFT "graftingthrough" polymerization using the electrodeposited G1CzMA onto gold surfaces. Note: formation of loops may also be possible in real systems.

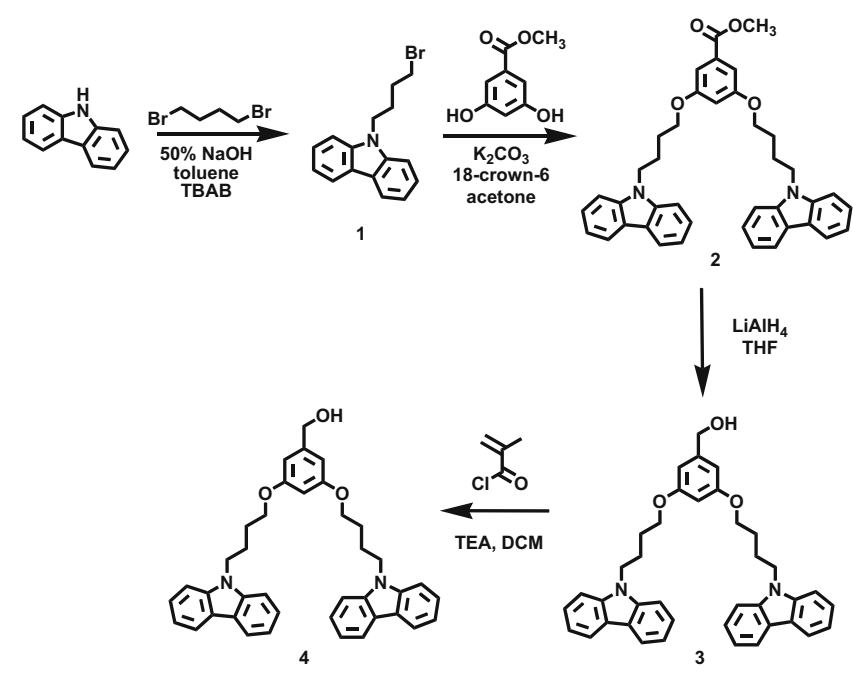

Fig. 2. Protocol for G1CzMA synthesis.

The study began with the synthesis of the monomer, G1CzMA (fig. 2). The synthesis was accomplished by the incorporation of the methacrylate unit to the carbazole dendron focal point by reacting the previously reported $\mathrm{G} 1 \mathrm{CzOH}$ dendron [44] with methacryloyl chloride under basic conditions. The esterification reaction is achieved in acceptable yield and almost no trace impurities are detectable after ${ }^{1} \mathrm{H}-\mathrm{NMR}$ analysis.

\subsection{Electrodeposition of G1CzMA}

The fabrication of the gold-coated glass substrate modified with electrodeposited G1CzMA is illustrated in fig. 1. The deposition was accomplished by potentiodynamic method. Because of the electroactive carbazole units, electrodeposition of the methacrylate unit is possible. This technique has also been reported by our group in previous studies toward the formation of $\mathrm{CPNs}$ and electrochemical crosslinking of dendrimers, hence an analogous approach was employed $[37,44]$. A scan rate of $100 \mathrm{mV} / \mathrm{s}$ was chosen after several experiments (not shown) of using different scan rates $(25,50$ and $100 \mathrm{mv} / \mathrm{s})$ where the highest current value was obtained using a $100 \mathrm{mV} / \mathrm{s}$ as scan rate. 


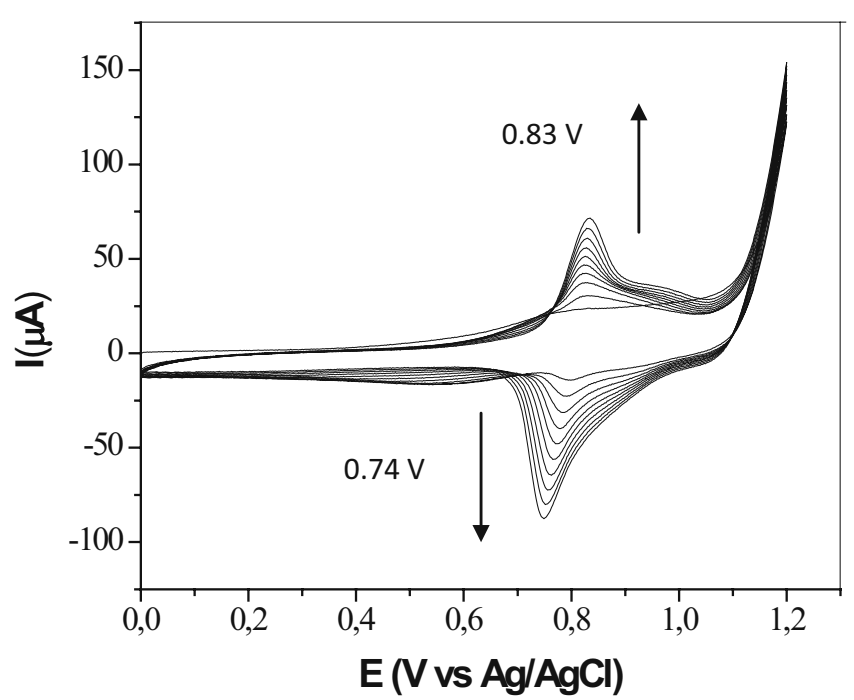

Fig. 3. Cyclic voltammogram of $5 \mathrm{mM}$ G1CzMA with $0.1 \mathrm{M}$ TBAH in THF. The potential was swept from 0 to $1200 \mathrm{mV}$ against an $\mathrm{Ag} / \mathrm{AgCl}$ reference electrode.

Table 1. The anodic and cathodic peak potentials, currents, peak separation, and the corresponding onset of the oxidation waves during the electrodeposition of G1CzMA.

\begin{tabular}{cccccc}
\hline Onset $(\mathrm{V})$ & $E_{\mathrm{pa}}(\mathrm{V})$ & $E_{\mathrm{pc}}(\mathrm{V})$ & $\Delta E(\mathrm{~V})$ & $i_{\mathrm{pa}}(\mu \mathrm{A})$ & $i_{\mathrm{pc}}(\mu \mathrm{A})$ \\
\hline 0.56 & 0.83 & 0.74 & 0.09 & 70.8 & -86.2 \\
\hline
\end{tabular}

The resulting crosslinked film is highly robust, stable and insoluble in many organic solvents.

The CV voltammogram of G1CzMA shows the onset of the carbazole oxidation at around $0.76 \mathrm{~V}$ on the first cycle (fig. 3). With the increasing number of cycles, an increasing current of oxidation waves from 0.7 to $0.9 \mathrm{~V}$ was observed, originating from the doping of the oligoor polycarbazoles, which also corresponded to the formation of polaronic and bipolaronic species. The reduction peaks from 1.2 to $0.7 \mathrm{~V}$ represented the de-doping process, in which the polarons and bipolarons gained electrons to give the neutral oligo- or polycarbazoles. The increase in current upon further cycling indicates a continuous mass deposition of the polymers and built up of more charges in the electropolymerized film. Carbazole-based polymers for the fabrication of electrochromic devices highly rely on this electrochemically induced doping-dedoping property. Table 1 summarizes the CV results based on the 10th cycle, including the onset of the polymer oxidation, anodic and cathodic peak potentials $\left(E_{\mathrm{pa}}\right.$ and $\left.E_{\mathrm{pc}}\right)$, and peak separation $(\Delta E)$ as well as the anodic and cathodic currents $\left(i_{\mathrm{pa}}\right.$ and $\left.i_{\mathrm{pc}}\right)$. According to the $i_{\mathrm{pa}} / i_{\mathrm{pc}}$ ratio, the $\mathrm{CV}$ waves were reversible.

The oxidation of polymeric carbazole typically appears in the second cycle. The peak of the oxidation potential shifted to higher values as the thickness of the film increased. This potential shift is attributed to the heterogeneous electron-transfer kinetics, IR drop of the film $\left(V_{\text {total }}=V_{\text {across film }}+I\left(R_{\text {film }}+R_{\text {soln }}\right)\right)$, and the decrease of the film conductivity [23]. The CV exhibited a re-

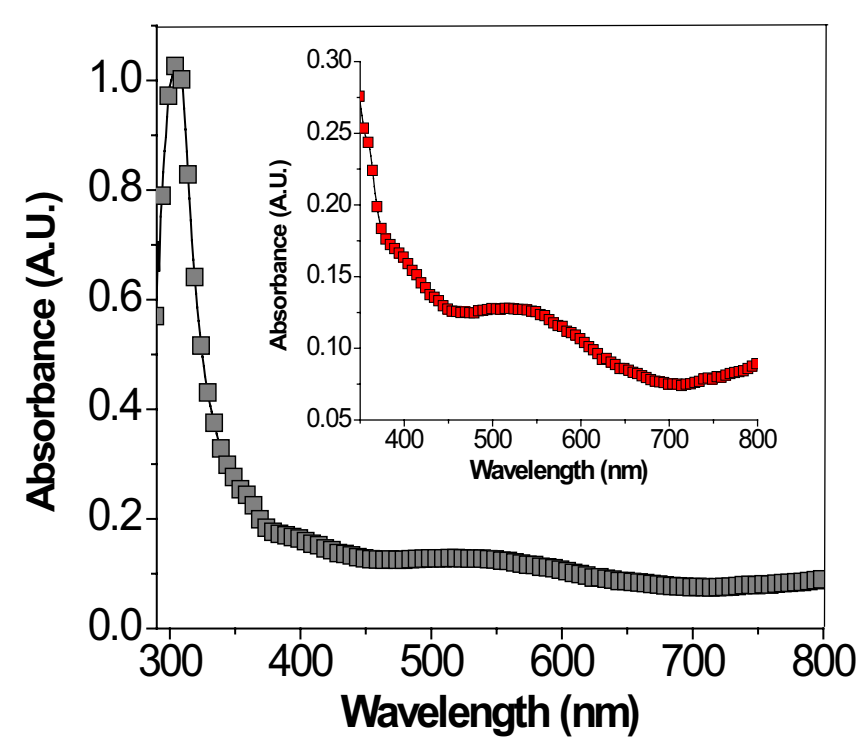

Fig. 4. UV-Vis spectrum of the electrodeposited G1CzMA film an ITO-coated glass. (inset: blow-up of the shoulder at 450-650 $\mathrm{nm}$ region to emphasize the presence of the peak. The peak is not too strong as expected for ultrathin films).

versible process, with an almost constant current increase after each cycle. The concentration of the monomer solution was also adjusted in several experiments $(0.5 \mathrm{mM}$, $2.5 \mathrm{mM}$ and $5.0 \mathrm{mM}$ ) but the most homogeneous surface was achieved using $5.0 \mathrm{mM} \mathrm{G1CzMA}$ solution in dry THF as observed by ellipsometry, AFM, and water contact angle. Also, this concentration was found to be necessary in order to have the appropriate amount of monomers available for RAFT "grafting-through" polymerization. With less than 10 cycles, almost no increase in thickness was detected by ellipsometry, whilst with more than 10 , very high thicknesses were observed, with RAFT polymerization impeded. For that reason, the best number of cycles for the electrodeposition process was limited to no more than 10, since the thickness obtained in this number of cycles was found to be the most appropriate for the grafting of polymer chains onto the surface. The thickness added after the electrodeposition of the G1CzMA under these conditions was $15.39 \pm 1.12 \mathrm{~nm}$. The static water contact angle was $75^{\circ} \pm 2^{\circ}$, showing a good film homogeneity.

As a control experiment, the same CV conditions were applied using methyl methacrylate instead of G1CzMA, to check if the double bond is affected under the application of the specified voltage. No anodic or cathodic peaks were observed in the control experiment (data not shown), demonstrating that the methacrylate moiety is preserved during the electrodeposition process and could readily be used for the next "grafting-through" process.

The spectroscopic property of the electrodeposited G1CzMA was also observed using UV-Vis spectroscopy. Figure 4 shows the UV-Vis spectrum of the electrogenerated G1CzMA after CV on ITO-coated glass using identical conditions with the gold as substrate (i.e. sweeping the potential from 0 to $1200 \mathrm{mV}$ vs. $\mathrm{Ag} / \mathrm{AgCl}$ non-aqueous reference electrode, 10 cycles, $5 \mathrm{mM}$ of 
Table 2. Conversions, theoretical molecular weights, actual molecular weights and PDI values of the free PMMA polymers formed in solution at different time intervals.

\begin{tabular}{ccccc}
\hline time $(\mathrm{h})$ & conversion $(\%)^{\mathrm{a}}$ & $M_{n}$, theory $^{\mathrm{b}}$ & $M_{n}, \mathrm{GPC}$ & $\mathrm{PDI}$ \\
\hline 1 & 2.92 & 2000 & 5123 & 1.15 \\
2 & 11.34 & 5707 & 9876 & 1.17 \\
4 & 39.57 & 19741 & 18567 & 1.18 \\
7 & 63.78 & 32359 & 32133 & 1.18 \\
8 & 78.98 & 39900 & 40678 & 1.19 \\
\hline
\end{tabular}

a Conversion was determined by NMR.

b $M_{n}$, theory $=M_{\text {monomer }} \times$ conv $\times[M]_{\mathrm{o}} \times[\mathrm{CTA}]_{\mathrm{o}}$.

G1CzMA in dry THF, and $0.1 \mathrm{M}$ of the supporting electrolyte TBAH). The absorption spectra of the film obtained after electropolymerization depicted a $\pi-\pi^{*}$ transition between $450-650 \mathrm{~nm}$, attributed to the formation of a $\pi$-conjugated polymer on the ITO substrate $[37,52,53]$.

\section{2 "Grafting through" of polymers on the surface}

To probe the effectiveness of the G1CzMA-modified Au surfaces for grafting polymers from a RAFT solution polymerization procedure, three different monomers were employed under typical homogeneous RAFT polymerization conditions. The monomers include styrene (S), methyl methacrylate (MMA) and tert-butyl acrylate (TBA). The molar ratio between the monomer, AIBN as the free radical initiator and the CTA 1 were 400:0.2:1. The reaction was first attempted using MMA as the monomer. The reaction was run for 8 hours at $60^{\circ} \mathrm{C}$. From a concentration of $8.32 \mathrm{mM}$ MMA, the brush thickness was observed to increase from 15.39 to $35.66 \mathrm{~nm}$, indicating a thickness of $20.27 \mathrm{~nm}$ for the grafted PMMA layer on the surface. Water contact angle of the PMMA film changed from $\sim 75^{\circ}$ to $\sim 55^{\circ}$, which are comparable to the literature values of the PMMA films on surfaces grown via surface-initiated RAFT polymerization $[54,55]$.

In order to estimate the molecular weight, $M_{n}$, and polydispersity index (PDI) of the grafted PMMA on the surface, the isolated sacrificial polymers were directly analyzed by GPC at different time intervals. Table 2 summarizes the results of the GPC analysis of the "free polymers" in solution. Figure 5 shows the GPC chromatograms of the PMMA formed at different time interval. The chromatograms were observed to be narrow and monomodal over the range of conversions investigated in this study, demonstrating good control of the polymerization of the monomers in solution under these conditions. With these results, the kinetic activity for the MMA polymerization was also investigated. Aliquots were taken from the reaction vessel at specific time intervals to monitor the evolution of $M_{n}$ and PDI as a function of monomer conversion (fig. 6) and the monomer conversion vs. time (fig. 7). As shown in fig. 6 , the polymerization of MMA showed the characteristics of a well-controlled living polymerization with a constant radical concentration as indicated by the linearity of plot between $\ln ((1 / 1-$ conv $))$ and the time

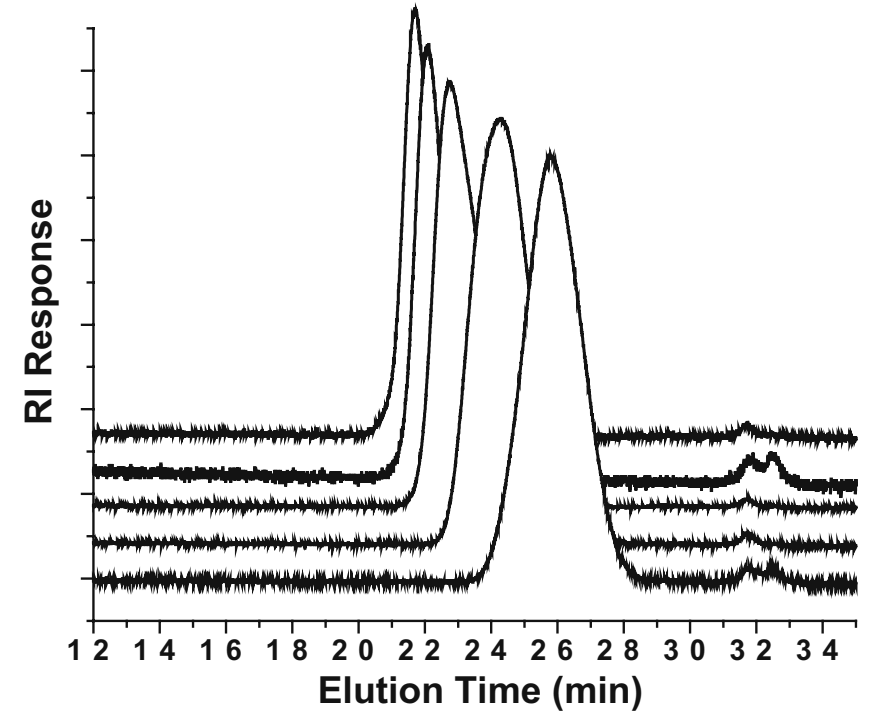

Fig. 5. GPC chromatograms collected over time for the polymerization of methyl methacrylate.

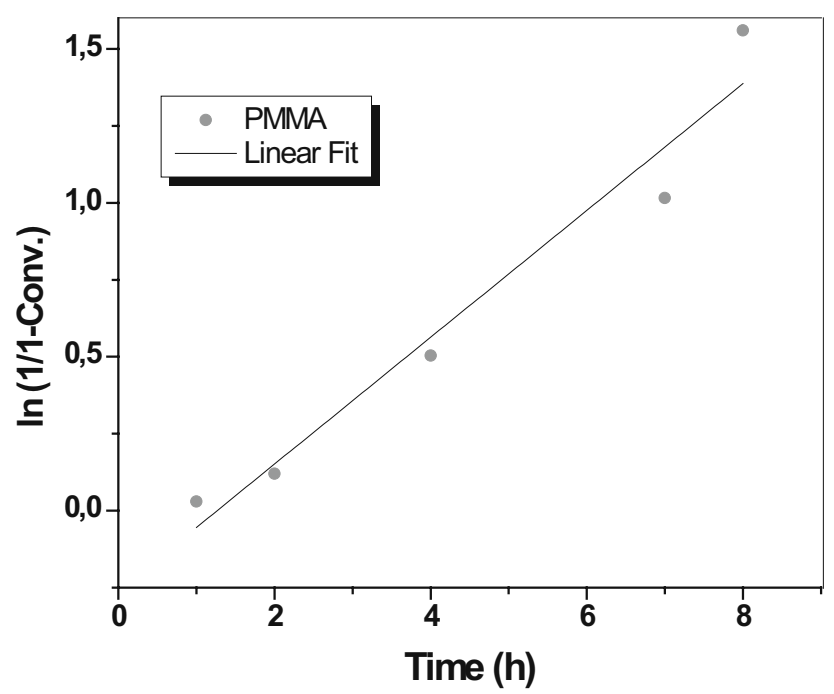

Fig. 6. Kinetic plot for the polymerization of methyl methacrylate monomer.

of polymerization. The controlled nature of the polymerization is further demonstrated by the linear increase in $M_{n}$ vs. conversion as shown in fig. 7. Furthermore, an excellent agreement between the experimentally determined $M_{n}$ (GPC) and the theoretical $M_{n}$ was observed (table 3 ), indicating that the CTA remained essentially intact and well-behaved over the course of the grafting-through polymerization under the described conditions, which should also allow the synthesis of well-defined polymers attached onto the surface.

For direct GPC analysis of polymers from surfaces, future work should include grafting-through polymerization on high surface to volume spherical particles followed by the direct degrafting of the polymer chains. Because particles have higher superficial areas, this should render more 


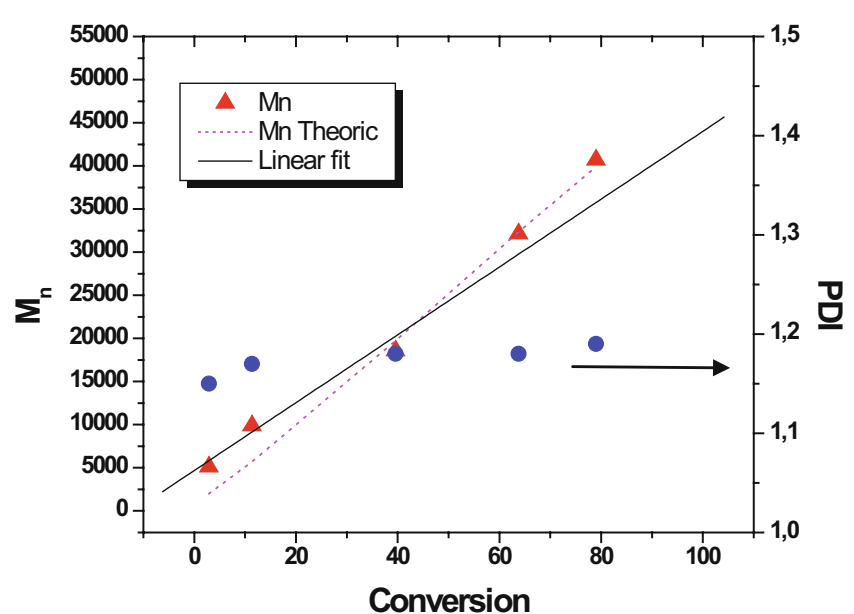

Fig. 7. Evolution of molecular weight and PDI with conversion for PMMA synthesis.

Table 3. Molecular weights and PDIs of the free polymers formed in solution as determined by GPC.

\begin{tabular}{|c|c|c|c|}
\hline Polymer & theor $M_{n}(\mathrm{~g} / \mathrm{mol})$ & $\operatorname{expt} M_{n}(\mathrm{~g} / \mathrm{mol})$ & PDI \\
\hline $\mathrm{PMMA}^{\mathrm{a}}$ & 39900 & 40678 & 1.19 \\
\hline $\mathrm{PS}^{\mathrm{b}}$ & 10015 & 10080 & 1.09 \\
\hline
\end{tabular}

a Synthesized using a monomer: CTA ratio of 400:1, and CTA: initiator (AIBN) 5:1, 8 hours of reaction, in THF: Benzene $1: 1$ at $60{ }^{\circ} \mathrm{C}$.

b Synthesized using a molar ratio between monomer: CTA 400:1, CTA: initiator(AIBN) 5:1, 12 hours of reaction, THF: benzene as solvent, $60^{\circ} \mathrm{C}$.

polymer chains attached onto the surface. Degrafting of polymer chains will allow enough polymers in solution to be analyzed by GPC. An important question is how to have the same or analogous electropolymerized monomers on the surface of the spherical particles as reported here.

XPS analysis of each surface modification step was also done to characterize the films produced. Changes in the intensity of the $\mathrm{C} 1 s, \mathrm{O} 1 s$, and $\mathrm{N} 1 s$ peaks, as well as the comparison of the apparent atomic percentages were analyzed to monitor the success of each modification step. The actual atomic percentages and theoretical atomic percentages are tabulated in table 4 for all the polymers grafted on the electrodeposited G1CzMA. In the case of the electrodeposited G1CzMA, the expected elemental peaks showed a good correlation of the composition with the theoretical values, confirming the modification of the gold surface with the electroactive monomer. A successful RAFT mechanism requires that all polymer chains be initiated at the same time, enabling a good grafting density. This would be desirable in order to have a better statistical probability of the growing radical chains finding the methacrylate unit at the surface of the electropolymerized film. Likewise, a good relative agreement was also observed with the polymers grafted on the surface. The slight variations of the actual percentages from the theoretical percentages can be explained by the fact that the actual percentages are taken from the polymers grafted on top of an underlying electrodeposited layer, which is different from the theoretical composition that does not take into account this underlying layer. Depending on the thickness or grafting density, the underlying layer could still give a contribution to the readings of the polymer on the surface. Furthermore, the incorporated CTA units are not accounted for in the theoretical percentages. The lower atomic percentages of $\mathrm{N} 1 s$ of the films after the grafting-through process as compared to the electrodeposited G1CzMA further confirms the tethering of the polymers on top of the electrodeposited layer. Another important observation is the presence of the sulfur on the surface after the grafting-through process, which signifies the occurrence of RAFT polymerization through the surface. The relatively small amount of sulfur on the surfaces of block copolymers is due to the relatively thicker films of the polymer formed, making the ratio of the sulfur lower than either the carbon or oxygen. It is assumed that there is only one CTA moiety for every polymer chain formed, no matter how long it is. It can be also noted that there is an increase in the $\mathrm{O} / \mathrm{C}$ ratio of the PMMA film as compared to the electrodeposited G1CzMA. For the PS surface, on the other hand, a high percentage of $\mathrm{C} 1 s$ is evident, that signifies the incorporation of a carbon-rich layer.

One of the major advantages of living radical polymerization, such as RAFT polymerization, is its ability to produce block copolymers to produce a more complex architecture on the surface. In this study, the PMMA and PS-modified surfaces were used as the macro-CTAs for the polymerization of the next blocks PS and PTBA, respectively. The second blocks were chosen according to their relative reactivity with the first block. Succeeding diblock copolymerization had to be performed with a monomer less reactive than the first block in order to successfully demonstrate the RAFT process as expected for a normal RAFT polymerization. The ability of the modified surfaces to form block copolymers on the surface was also verified by XPS. Table 4 shows a corresponding increase in the $\mathrm{C} 1 s$ percentage and a decrease of the $\mathrm{O} 1 s$ upon the formation of the PMMA- $b$-PS block. This is in accordance with the expected addition of the PS chains on top of the surface, corresponding to the second block. On the other hand, the PS-b-PTBA block showed a decrease in the $\mathrm{C} 1 s$ composition and an increase in $\mathrm{O} 1 s$ percentage owing to the growth of PTBA block on top of the PS layer.

Figure 8 further displays the formation of the diblock copolymer (only the PMMA- $b$-PS is shown for simplicity). Qualitatively, the comparison in the intensities of the peak can be easily assessed in the spectra where an increase in the $\mathrm{O} / \mathrm{C}$ ratio is observed upon the formation of the PMMA film, owing to the oxygen contributed by the grafted PMMA. O/C ratio was then decreased after the carbon-rich PS block was grafted on the surface. The disappearance of the $\mathrm{N} 1 s$ peak attributed to the carbazole nitrogen of G1CzMA is also noticeable after the grafting of the polymers (PMMA and PMMA- $b$-PS), denoting the formation of a thick polymer film on top of the surface that attenuated the $\mathrm{N} 1 s$ signal of the underlying G1CzMA. 
Table 4. Actual atomic percentage of the modified gold substrates from XPS. Note: the XPS polymer brush layers contain possible percentage composition that includes the underlying electrodeposited C1CzMA layer. The theoretical XPS composition of the polymer brush layers does not include the underlying electrodeposited C1CzMA layer. Calculations for theoretical composition discount for the $\mathrm{H}$ atom in each compound. S $2 p$ concentration is not given in the theoretical calculations since it only comes from the CTA attached on the grafted polymer chain. N.A. = not applicable (calculation of theoretical composition was not done due to the complexity of the system).

\begin{tabular}{cccccccc}
\hline \multirow{2}{*}{ Surface } & \multicolumn{3}{c}{ Actual atomic percentages } & \multicolumn{3}{c}{ Theoretical atomic percentages } \\
\cline { 2 - 7 } & $\mathrm{C} 1 s$ & $\mathrm{O} 1 s$ & $\mathrm{~N} 1 s$ & $\mathrm{~S} 2 p$ & $\mathrm{C} 1 s$ & $\mathrm{O} 1 s$ & $\mathrm{~N} 1 s$ \\
\hline G1CzMA & 86.5 & 9.9 & 3.7 & 0 & 83.9 & 11.2 & 4.9 \\
PMMA & 73.9 & 25.9 & $<0.1$ & 0.2 & 65.2 & 34.8 & 0 \\
PS & 92.7 & 6.8 & $<0.1$ & 0.5 & 100 & 0 & 0 \\
PMMA- $b$-PS & 80.4 & 19.6 & $<0.1$ & $<0.1$ & N.A. & N.A. & 0 \\
PS- $b$-PTBA & 90.8 & 9.2 & $<0.1$ & $<0.1$ & N.A. & N.A. & 0 \\
\hline
\end{tabular}

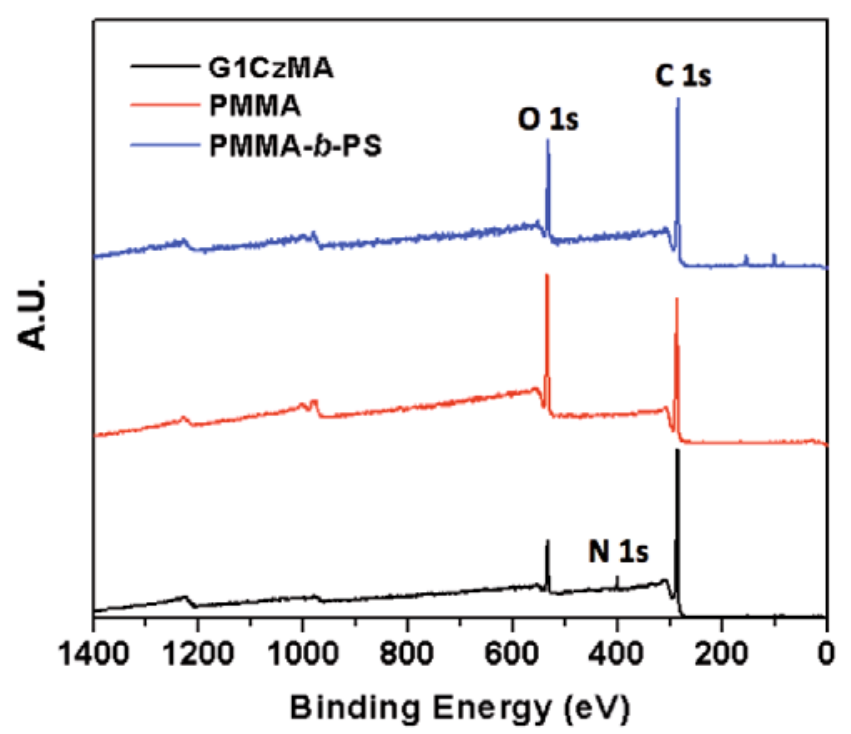

Fig. 8. Wide-scan XPS spectra of G1CzMA (black line), PMMA homopolymer film (red line), and PMMA-b-PSty diblock copolymer (blue line) grafted on Au surface.

These XPS results were further supported by the ellipsometric thickenesses and water contact angles of the films (table 5). Thickness and contact angle data show the incorporation of the materials during each succeeding steps. For instance, the PMMA-modified surface showed an increase of $\sim 20 \mathrm{~nm}$ from the electrodeposited G1CzMA while an increase of about $11 \mathrm{~nm}$ was added after block copolymerization with PS. These results were verified by the contact angle data that showed a decrease during the modification of G1CzMA layer with the relatively more hydrophilic PMMA layer and an increase again in the water contact angle value after grafting the second block of the hydrophobic PS. A homogeneous PS film has a value of about $95^{\circ}$, which indicates that these films are far from being a homogeneous PS film layer, but is largely of lower grafting density of the PS block. This result is expected because of the lesser accessibility of the CTA end group that may be buried in the layer of the first block.
Similar results were also observed for the PS and PS- $b$ PTBA block where thickness of $\sim 9 \mathrm{~nm}$ was added to the G1CzMA layer after grafting through of PS and additional $\sim 22 \mathrm{~nm}$ was incorporated after the second block of PTBA was grafted. Water contact angle measurements showed a change from $90^{\circ}$ to $82^{\circ}$, indicating the presence of a less hydrophobic character on top of the surface.

The morphological changes of the films after every modification step were also investigated. According to the desired application of the ultrathin film, it is very important to determine the relative smoothness and homogeneity of the films. The film growth and morphology changes were investigated by AFM using an intermittent contact mode (fig. 9). Figure 9a shows the morphology of the film obtained after the electrodeposition of the G1CzMA. A homogeneous and morphologically continuous electrodeposited film was formed on the substrate. The film is uniform throughout the substrate, with very small domain sizes. After the PMMA homopolymer synthesis (fig. 9b) a change in the morphology can be observed. Elongated structures on the morphology with high regularity and homogeneity are evident. The surface is almost completely covered by the material as also probed by ellipsometry, after sampling across the sample. Figure 9c shows the morphology of the diblock copolymer PMMA- $b$-PS, which showed larger globular domains with relatively good regularity. All these images were obtained as-is after washing and Soxhlet extraction procedure. Annealing with different solvents or temperatures should allow formation of various equilibrated morphologies.

\section{Conclusions}

We have demonstrated a novel route to anchor polymer chains onto a surface via electrodeposition of an electroactive methacrylate dendron through CV with subsequent polymerization of monomers such as methacrylates, styrenes and acrylates from solution in a RAFT graftingthrough approach. The process was shown to be versatile and efficient in preparing homopolymers and two different diblock copolymers with good homogeneity, and good control over the polymerization mechanism as evidenced 
Table 5. Thickness and contact angle data for the homopolymers and diblock copolymer films on the surface.

\begin{tabular}{lcc}
\hline \multicolumn{1}{c}{ Surface } & $\Delta$ thickness $(\mathrm{nm})^{\mathrm{a}}$ & ${\text { Water contact angle data }(\theta)^{\mathrm{b}}}^{\mathrm{b}}$ \\
\hline G1CbzMA & $15.39 \pm 1.12$ & $75.6 \pm 1.2$ \\
PMMA & $20.27 \pm 5.92$ & $55.3 \pm 3.4$ \\
PMMA- $b$-PSty & $11.32 \pm 1.03$ & $82.0 \pm 0.6$ \\
PSty & $9.16 \pm 3.11$ & $90.2 \pm 1.1$ \\
PSty-b-PTBA & $21.6 \pm 1.12$ & $82.9 \pm 4.8$ \\
\hline
\end{tabular}

\footnotetext{
a Thicknesses were determined by ellipsometry and are an average of five samplings across the sample. b Static water contact angle measurements were taken across the sample and are an average of five measurements.
}
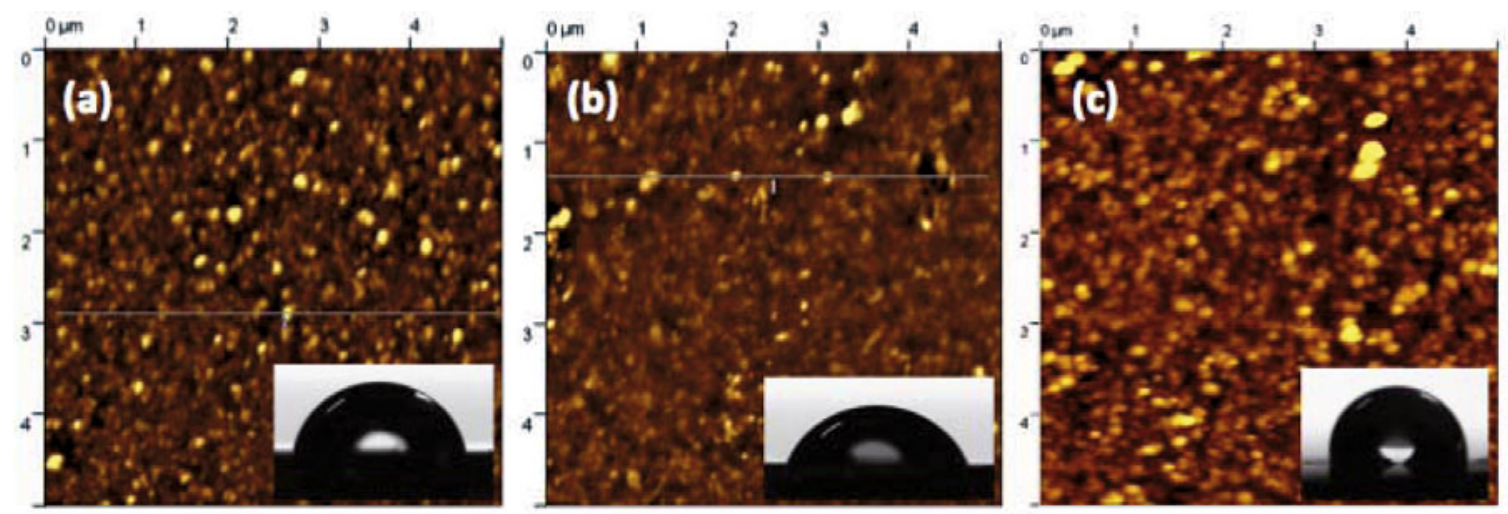

Fig. 9. AFM topography images of the gold surface (a) after electropolymerization of G1CzMA, (b) after the grafting through of the PMMA layer, and (c) after the diblock formation of PMMA-b-PS. (Inset: water contact angles of (a) G1CzMA, (b) PMMA film, and (c) PMMA-b-PS diblock copolymer on Au surface.)

by the monomodal GPC traces and the uniformity of the film morphologies by AFM. Several characterization techniques including ellipsometry, static contact angle measurements, XPS, UV-Vis spectroscopy, and CV confirmed the electrodeposition of methacrylate dendron and the grafting of the polymer chains onto the surface. In the future, it will be possible to focus on the formation of other types of electropolymerizable monomers and subsequently conducting polymers in combination with grafted polymers on surfaces.

This work was partially supported by the National Science Foundation ARRA-CBET-0854979, DMR-10-06776, Texas NHARP 01846, and the Robert A. Welch Foundation, E-1551. Technical support from Viscotek Inc., KSV Instruments, Agilent Technologies, is also appreciated. C.D. Grande thanks the Instituto Colombiano de Investigaciones Científicas, COLCIENCIAS, for economic support through a doctoral fellowship and also to the CENM, Centro de Excelencia en Nuevos Materiales for economic support.

Open Access This article is distributed under the terms of the Creative Commons Attribution Noncommercial License which permits any noncommercial use, distribution, and reproduction in any medium, provided the original author(s) and source are credited.

\section{References}

1. R.C. Advincula, W.J. Brittain, K.C. Caster, J. Ruhe, Polymer Brushes: Synthesis, Characterization, Applications (Wiley-VCH, New York, 2004).

2. L.F. Thompson, C.G.M.J. Bowden, Introduction to Microlitography, 2nd edition (Willson, American Chemical Society, Washington DC, 1994).

3. J. Ruhe, V. Novotny, T. Clarke, G.B. Street, J. Tribol. Trans. ASME 118, 663 (1996).

4. M. Tirrel, E. Kokkoli, M. Biesalski, Surf. Sci. 500, 61 (2002).

5. J.P. Andrade, Surface and Interfacial Aspects of Biomedical Polymers (Plenum Press, New York, 1985).

6. W.A. Levinson, A. Arnold, O. Dehodgins, Polym. Eng. Sci. 33, 980 (1993).

7. P. Yimsiri, M.R. Mackley, Chem. Eng. Sci. 61, 3496 (2006).

8. G. Decher, Science 277, 1232 (1997).

9. J. Park, S. Lee, H. Lee, Org. Electron. 7, 256 (2006).

10. S. Minko, S. Patil, V. Datsyuk, F. Simon, K.J. Eichhorn, M. Motornov, D. Usov, I. Tokarev, M. Stamm, Langmuir 18, 289 (2002).

11. B. Zhao, W.J. Brittain, Prog. Polym. Sci. 25, 677 (2000).

12. J. Pyun, T. Kowalewski, K. Matyjaszewski, Macromol. Rapid. Commun. 24, 1043 (2003).

13. K.P. Krenkler, R. Laible, K. Hamann, Angew. Makrom. Chem. 53, 101 (1953). 
14. A.V. Dmitrenko, N.E. Shadrina, S.S. Ivanchev, N.N. Ulinskaya, A.M. Volkov, J. Chromatogr. 520, 21 (1990).

15. K. Hashimoto, T. Fujisawa, M. Kobayashi, R. Yosomiya, J. Macromol. Sci.-Chem. A18, 173 (1982).

16. K. Hashimoto, T. Fujisawa, M. Kobayashi, R. Yosomiya, J. Appl. Polym. Sci. 27, 4529 (1982).

17. V.I. Trachenko, Y.N. Zil'berman, T.F. Shatskaya, E.G. Pomerantseva, Polym. Sci., USSR 28, 646 (1986).

18. N.B. Zhitenev, A. Sidorenko, D.M. Tennant, R.A. Cirelli, Nat. Nanotech. 2, 237 (2007).

19. S.Y. Jang, G.A. Sotzing, Macromolecules 37, 4351 (2004).

20. G. Zotti, R.A. Marin, M.C. Gallazzi, Chem. Mater. 9, 2945 (1997).

21. S.Y. Jang, G.A. Sotzing, M. Marquez, Macromolecules 35, 7293 (2002)

22. N. DiCesare, M. Belletete, C. Marrano, M. Leclerc, G. Durocher, J. Phys. Chem. A 103, 795 (1999).

23. P. Taranekar, A. Baba, T. Fulghum, R. Advincula, Macromolecules 38, 3679 (2005).

24. C.J. Xia, R.C. Advincula, Macromolecules 34, 5854 (2001).

25. A. Baba, K. Onishi, W. Knoll, R.C. Advincula, J. Phys. Chem. B 108, 18949 (2004).

26. P. Taranekar, A. Baba, T.M. Fulghum, R. Advincula, Macromolecules 38, 3679 (2005).

27. T. Fulghum, S.M.A. Karim, A. Baba, P. Taranekar, T. Nakai, T. Masuda, R.C. Advincula, Macromolecules 39, 1467 (2006).

28. P. Waenkaew, P. Taranekar, P. Phanichphant, R. Advincula, Macromol. Rapid Commun. 28, 1522 (2007).

29. B. Romero, M. Schaer, M. Leclerc, D. Ades, A. Siove, L. Zuppiroli, Synth. Met. 80, 271 (1996).

30. Z.H. Peng, Z.N. Bao, M.E. Galvin, Chem. Mater. 10, 2086 (1998).

31. A. Van Dijken, J.J.A.M. Bastiaansen, N.M.M. Kiggen, B.M.W. Langeveld, C. Rothe, A. Monkman, I. Bach, P. Stossel, K. Brunner, J. Am. Chem. Soc. 126, 7718 (2004).

32. B. Kippelen, K. Tamura, N. Peyghambarian, A.B. Padias, H.K. Hall, Phys. Rev. B 48, 10710 (1993).

33. Y.D. Zhang, T. Wada, H. Sasabe, J. Mater. Chem. 8, 809 (1998).

34. I. Schwendeman, R. Hickman, G. Sonmez, P. Schottland, K. Zong, D.M. Welsh, J.R. Reynolds, Chem. Mater. 14, 3118 (2002).
35. D. Witker, J.R. Reynolds, Macromolecules 38, 7636 (2005).

36. Q.D. Ling, Y. Song, S.J. Ding, C.X. Zhu, D.S.H. Chan, D.L. Kwong, E.T. Kang, K.G. Neoh, Adv. Mater. 17, 455 (2005).

37. P. Taranekar, T. Fulghum, A. Baba, D. Patton, R. Advincula, Langmuir 23, 908 (2007).

38. C. Huang, G. Jiang, R. Advincula, Macromolecules 41, 4661 (2008).

39. Z. Peng, Z. Bao, M.E. Galvin, Chem. Mater. 10, 2086 (1998).

40. B. Liu, W.L. Yu, Y.H. Lai, W. Huang, Chem. Mater. 13, $1984(2001)$

41. C. Xia, X.W. Fan, M.K. Park, R.C. Advincula, Langmuir 17, 7893 (2001).

42. P. Taranekar, X.W. Fan, R. Advincula, Langmuir 18, 7943 (2002).

43. P. Taranekar, J.Y. Park, T. Fulghum, D. Patton, R. Advincula, Adv. Mater. 18, 2461 (2006).

44. P. Taranekar, T. Fulghum, D. Patton, R. Ponnapati, G. Clyde, R. Advincula, J. Am. Chem. Soc. 129, 12537 (2007).

45. J. Chiefari, Y. Chong, F. Ercole, J. Krstina, J. Jeffrey, T. Le, R. Mayadunne, G. Meijs, C. Moad, G. Moad, E. Rizzardo, S. Thang, Macromolecules 31, 5559 (1998).

46. Z. Bo, W. Zhang, X. Zhang, C. Zhang, J. Shen, Macromol. Chem. Phys. 199, 1323 (1998).

47. Y. Fu, Y. Li, J. Li, S. Yan, Z. Bo, Macromolecules 37, 6395 (2004).

48. H.G. Tompkins, W.A. McGahan, Spectroscopic Ellipsometry and Reflectometry (John Wiley \& Sons Inc., USA, 1999).

49. K. Matyjaszewski, T.P. Davis, Handbook of Radical Polymerization (Wiley-Interscience, New York, 2002).

50. J. Raula, J. Shan, M. Nuopponen, A. Niskanen, H. Jiang, E.I. Kauppinen, H. Tenhu, Langmuir 19, 3499 (2003).

51. C. McCormick, A. Lowe, Acc. Chem. Res. 37, 312 (2004).

52. T.M. Fulghum, P. Taranekar, R. Advincula, Macromolecules 41, 5681 (2008).

53. S. Hayashida, K. Sukegawa, O. Niwa, Synth. Met. 35, 253 (1990).

54. M.H. Stenzel, L. Zhang, W.T.S. Huck, Macromol. Rapid Commun. 27, 1121 (2006).

55. R. Ranjan, W.J. Brittain, Macromol. Rapid Commun. 28, 2084 (2007) 